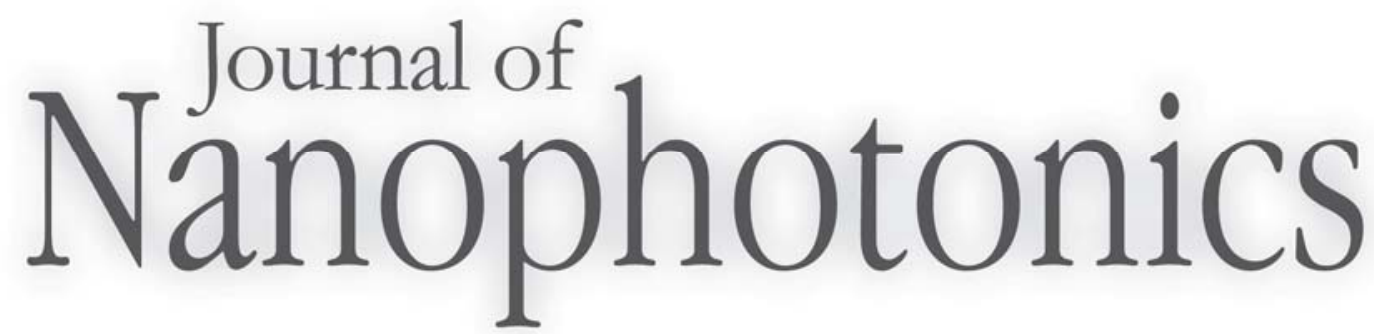

SPIEDigitalLibrary.org/jnp

\title{
Book Review: \\ Principles of Nanophotonics
}

Reviewed by Tom G. Mackay

\section{O SPIE}




\section{BOOK REVIEW}

\section{Principles of Nanophotonics}

Motoichi Ohtsu, Kiyoshi Kobayashi, Tadashi Kawazoe, Takashi Yatsui and Makoto Naruse, 228 pages, ISBN 978-1-58488-972-4, CRC Press, Boca Raton, FL, USA (2008), US\$ 79.95, hardcover.

Reviewed by Tom G. Mackay, University of Edinburgh, School of Mathematics and Maxwell Institute for Mathematical Sciences, Edinburgh EH9 3JZ, United Kingdom, T.Mackay@ed.ac.uk.

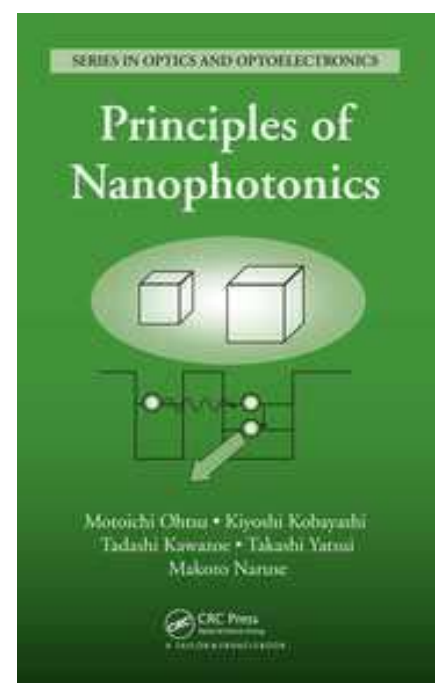

Taxonomy can present knotty issues, especially during the early phases of a rapidly developing discipline. The authors of Principles of Nanophotonics draw a sharp distinction between 'conventional plasmonics' (including topics such as photonic crystals, plasmonics and metamaterials) and 'nanophotonics' which they perceive as the study of optical near-field effects that allow the diffraction limit to be transcended. The latter but not the former is covered in this book. This rather narrow classification of 'nanophotonics' is not universally shared - others take a broader perspective, more in line with the scope of the Journal of Nanophotonics.

In five chapters, Principles of Nanophotonics delivers an overview of certain optical near-field effects and their applications, concentrating primarily on the authors' own research. Although it is not explicitly stated, the target audience would appear to be graduate students (but no problems are provided) and researchers in nanophotonics. The opening chapter sets the stage nicely for the chapters which follow, by briefly surveying historical aspects as well as highlighting areas where applications of optical near-field effects are being usefully pursued at present. The second chapter is heavy on theory. A quantum mechanical description of the optical near-field interaction between nanoscale particles in close proximity is presented. This approach is claimed to be more physically intuitive than the more conventional classical optics approach. Nanophotonic devices, most notably AND and NOT gates, are described in detail in the third chapter. The fourth chapter deals with nanophotonic fabrication techniques, including photochemical vapour deposition, photolithography and self-assembly methods. The final chapter represents the culmination of the preceding four chapters, wherein a methodological approach to nanophotonic systems is presented. Topics included here include parallel architectures, interconnections and hierarchies. Five appendixes supplement the theory presented in the second chapter.

There are several commendable aspects to this book. The five chapters hang together well to create a logical natural structure. Each chapter is lavishly illustrated with figures. As an informed and well-referenced commentary on the state-of-the art in optical near-field effects and their applications, it works admirably well. However, there some points which this reviewer feels could have been improved upon. The scope could have been broader. For example, some coverage of the increasingly important topic of nanobiophotonics would have made a valuable 
addition. There is a noticeable stylistic unevenness in the prose between the chapters, and in places the prose does not flow as fluently as might be expected. The mathematical notation adopted is not uniform throughout. Some of the figures would have benefitted from more detailed explanations, and some would have benefitted from being larger and incorporating colour. Some of the descriptions of nanophotonic devices and applications will soon be out of date (indeed, some may already be so by now) - of course, this should not be taken as a criticism of the book, as it is an inevitable consequence of the ongoing rapid progress being made in this area by the authors and others.

In summary, this book is reminiscent of the proverbial curate's egg: it provides an engaging overview, likely to be of some value to those active in research on near-field optics, although they may wish that the scope was broader and the presentation was more polished. 